\title{
MOVILIDAD TERRITORIAL, CIRCUITOS LABORALES Y DESIGUALDADES EN PRODUCCIONES AGRARIAS DE ARGENTINA: ABORDAJES INTERDISCIPLINARES Y DEBATES CONCEPTUALES
}

\author{
Territorial mobility, labor circuits and inequalities in agriculture \\ in Argentina: interdisciplinary approaches and conceptual debates
}

\author{
Verónica Trpin ${ }^{1}$ \\ Cynthia Pizarro ${ }^{2}$
}

\begin{abstract}
Resumen. Este artículo analiza aquellos cruces interdisciplinares entre la geografía, la sociología y la antropología que dieron lugar al diálogo entre los conceptos movilidad territorial y desigualdades de clase, género y razaetnia, especialmente en temáticas relacionadas con las diversas inserciones laborales de varones y mujeres migrantes en producciones agrarias en Argentina. Se señala la necesidad de considerar las complejidades de la categoría migrantes laborales y se reflexiona acerca de los desafíos que este tipo de abordajes plantean para el estudio de las movilidades territoriales, los mercados de trabajo y las desigualdades socio-culturales.

Palabras clave: movilidad territorial, circuitos laborales, trabajo rural, desigualdades, Argentina.

Abstract. This paper analyses the interdisciplinary crossroads between Geography, Sociology and Anthropology that have led to a dialogue between the concepts: territorial mobility and inequalities of class, gender and race-ethnicity, focusing on topics related to diverse insertions of male and female migrants in agriculture labor markets in Argentina. It remarks that the complexities of the category labor migrants should be considered and it reflects on the challenges that such approaches pose to the study of territorial mobilities, labor markets and socio-cultural inequalities.
\end{abstract}

Keywords: territorial mobility, labor circuits, rural work, inequalities, Argentina.

Consejo Nacional de Investigaciones Científicas y Técnicas (IPEHCS-CONICET), Universidad Nacional del Comahue (UNCo). Buenos Aires, Argentina.

2 Consejo Nacional de Investigaciones Científicas y Técnicas (CONICET), Universidad de Buenos Aires (UBA). Buenos Aires, Argentina. 


\section{Introducción}

Este artículo tiene el propósito de analizar algunas líneas de abordaje de las migraciones, observando los cruces interdisciplinares y los debates teóricos que permiten instalar conceptos como movilidad y territorio en diálogo con los de clase social, género y marcaciones étnico-racializantes, especialmente en temáticas relacionadas con diversas inserciones laborales de varones y mujeres migrantes en producciones agrarias en Argentina. En tal sentido, recientemente se ha señalado que estos/as trabajadores/as ocupan las posiciones más precarias que les son asignadas en las jerarquías de esos mercados de trabajo, debido a la manera en que se intersectan dichas desigualdades ${ }^{3}$ en los territorios por donde circulan ${ }^{4}$.

Consideramos relevante dar cuenta de la manera en que estos enfoques influyeron en los estudios migratorios en Argentina, a fin de remarcar la complejidad de la categoría migrantes laborales, profundizar temáticas pendientes y reflexionar sobre nuevos desafíos para el estudiode las movilidades territoriales, los mercados de trabajo y las desigualdades.

Diversos antecedentes provenientes de la geografía, la sociología y la antropología han abierto el camino para desentrañar las maneras en que se yuxtaponen las desigualdades de clase, de raza-etnia y de género en contextos de profunda precarización social como el trabajo de los y las migrantes en espacios rurales. Consideramos necesaria la recuperación de debates en torno al "trabajo desvalorizado" 5 en los procesos de reproducción del capital que son posibilitados por una significativa explotación de la mano de obra. Particularmente, nos focalizaremos en las maneras en que ciertas personas, definidas como "migrantes laborales", se articulan en mercados laborales agropecuarios en Argentina.

\section{Territorios y movilidad en el abordaje de las migraciones rurales}

En las últimas décadas, la incorporación de la mirada territorial en los estudios sobre estructuras agrarias ha influido en el abordaje de la incidencia de los procesos migratorios en los mercados laborales agropecuarios. En el marco del giro territorial tanto en el discurso científico como en los ámbitos políticos, la noción de territorio es parte de un nuevo "sentido común" desde el cual analizar e intervenir sobre el medio rural y su desarrollo.

\footnotetext{
ANTHIAS, Floya. The Material and the Symbolic In Theorizing Stratification: Issues Of Gender, Ethnicity and Class; CRENSHAW, Kimberlé. Mapping the Margins: Intersectionality, Identity Politics, and Violence Against Women of Color.

4 TARRIUS, Alain. Leer, describir, interpretar. Las circulaciones migratorias: conveniencia de la noción de "territorio circulatorio". Los nuevos hábitos de la identidad.

5 FALQUET, Jules. La règle du jeu: repenser la co-formation des rapports sociaux de sexe, de classe et de «race» dans la mondialisation néolibérale.
} 
En esta dirección, las investigaciones incorporaron (en diferentes medidas y con mayor o menor perspectiva crítica respecto de los cuerpos teóricos utilizados) aspectos vinculados con las dinámicas poblacionales, los modos de apropiación del espacio y los recursos, la circulación de capital y las relaciones de poder, para dar cuenta de los procesos de cambio y de las configuraciones sociales resultantes en Argentina ${ }^{6}$. Muchos de los trabajos más relevantes en este aspecto muestran influencias de las corrientes críticas de la geografía ${ }^{7}$, ya que problematizan la concepción de territorio y hacen especial hincapié en la necesidad de analizar las relaciones de poder implicadas en los procesos de configuración y transformación territorial.

Estos estudios plantean que el territorio es una construcción social en la que subyace el trabajo como actividad transformadora por excelencia que conduce a una artificialización constante de la dinámica "natural". Desde esta perspectiva, el trabajo es definitorio de la espacialidad en un contexto determinado, puesto que articula sus usos y la apropiación de los recursos que conllevan diferentes disputas y conflictos por su control. Desde esta perspectiva el territorio es considerado como una fuente de recursos en el marco de la relación capital/trabajo y como un producto de la división territorial del trabajo ${ }^{8}$.

En esta línea, los aportes de la geografía, en diálogo con los de la antropología, han enriquecido aquellos estudios que analizan las circulaciones de las poblaciones de un lado y otro de las fronteras ${ }^{9}$. Dichos abordajes atienden las maneras en que las culturas o "habitus migratorios" de las sociedades de origen modelan las motivaciones y las trayectorias migratorias ${ }^{10} \mathrm{e}$ indagan sobre las formas en que los migrantes construyen diversos territorios ${ }^{11}$. Así, los

6 Ver, entre otros autores, ALBANESI, Roxana, PREDA, Graciela. El enfoque territorial como propuesta de intervención para el desarrollo. Reflexiones desde una perspectiva latinoamericana; MANZANAL, Mabel. Regiones, territorios e institucionalidad del desarrollo rural; MANZANAL, Mabel. Territorio, poder e instituciones: una perspectiva crítica; MANZANAL, Mabel, ARQUEROS, María Ximena, ARZENO, Mariana, NARDI, María. Desarrollo territorial en el norte argentino. Una perspectiva crítica; REBORATTI, Carlos. El territorio rural: iactor o escenario?; SILI, Marcelo. La Argentina Rural. De la crisis de la modernización agraria a la construcción de un nuevo paradigma de desarrollo de los territorios rurales.

7 HAESBAERT, Rogerio. O mito da desterritorialização: do fim dos territórios à multiterritorialidade; HARVEY, David. El nuevo imperialismo: acumulación por desposesión; SANTOS, Milton. Espacio y método; MANCANO FERNANDES, Bernardo. Movimentos socioterritoriais e movimentos socioespaciais: contribuição teórica para uma leitura geográfica dos movimentos sociais.

8 HAESBAERT, op. cit.; RADONICH, Martha. Territorio, migración y trabajo en la fruticultura del norte de la Patagonia; RAFFESTIN, Claude. Por uma geografia do poder.

9 CAGGIANO, Sergio. Lo que no entra en el crisol. Inmigración boliviana, comunicación intercultural y procesos identitarios; GRIMSON, Alejandro. Fronteras, naciones e identidades: la periferia como centro; RADONICH, Martha, CIARALLO, Ana, TRPIN, Verónica. Chilenos y bolivianos en la conformación de territorios en áreas rurales del Alto Valle de Río Negro, Argentina.

10 RIVERO SIERRA, Fulvio. Condiciones de emigración y cultura migratoria en dos casos de Bolivia: Toropalca en Potosí y B Plan 3000 en Santa Cruz de la Sierra.

${ }^{11}$ BENENCIA, Roberto. Inserción de inmigrantes bolivianos y mercado de trabajo rural argentino. 
territorios comenzaron a ser analizados como una construcción de los grupos migrantes en el marco de sus procesos de movilidad sustentados en redes, y no como definiciones provenientes exclusivamente de los estados nacionales.

Los mercados de trabajo rurales de Argentina se caracterizaron históricamente por estar conformados por trabajadores estacionales o temporarios, con empleos precarios y organizados en territorios que incluyen áreas distantes, vinculadas a través de enganchadores ${ }^{12}$. Estas formas de contratación de la mano de obra procedente de diversas zonas del país o de países limítrofes fueron típicas en algunas economías regionales articuladas alrededor de actividades tales como la vendimia o la zafra de la caña de azúcar.

Tanto las migraciones internas como las internacionales en contextos agrarios fueron objeto de investigación desde la década del sesenta. Durante el último cuarto del siglo XX se estudiaron los procesos de movilidad de trabajadores hacia las grandes urbes en expansión ${ }^{13}$ y hacia producciones agrarias de alta demanda de mano de obra ${ }^{14}$.

En las últimas décadas, se ha investigado sobre los circuitos productivos rurales que demandan estacionalmente mano de obra para las cosechas, destacando la importancia de los procesos de movilidad territorial de los trabajadores en la configuración de territorialidades que trascienden las delimitaciones locales. Esos análisis retoman aportes de la geografía, al proponer una nueva forma de conceptualizar la migración a partir de la dimensión circulatoria, lo cual permite problematizar la cuestión de la direccionalidad

Familias transnacionales en la conformación de territorios productivos; BENENCIA, Roberto, ATAIDE, Soraya. Segmented Labor Market and Migratory Identity Constructions in Two Horticultural Areas in the Province of Salta; PIZARRO, Cynthia. Espacios socioculturales "bolivianos" trans-urbanos en el Área Metropolitana de Buenos Aires.

12 BENENCIA, Roberto, APARICIO, Susana (coords.). Nuevas formas de contratación en el trabajo agrario.

${ }^{13}$ BELVEDERE, Carlos, CAGGIANO, Sergio, CASARAVILLA, Diego et alii. Racismo y discurso: una semblanza de la situación argentina; FORNI, Floreal, ROLDÁN, Laura. Trayectorias laborales de residentes de áreas urbanas pobres. Un estudio de casos en el conurbano bonaerense; GERMANI, Gino. Emigración del campo a la ciudad y sus causas; ROFMAN, Alejandro. Economías regionales extrapampeanas y exclusión social en el marco del ajuste; SOCHACZEWSKI, Evelyn. Y salió a vender el día. Los desconocidos migrantes temporarios urbanos.

${ }^{14}$ BALAN, Jorge. Migraciones temporarias y mercado de trabajo rural en América Latina; BISIO, Raúl, FORNI, Floreal. Economía de enclave y satelización del mercado de trabajo rural. El caso de los trabajadores con empleo precario en un ingenio azucarero del noroeste argentino; CONTI, Viviana, LAGOS, Marcelo. Mano de obra indígena en los ingenios de Jujuy a principios de siglo; DANDLER, Jorge, MEDEIROS, Carmen. Temporary Migration from Cochabamba, Bolivia to Argentina: Patterns and Impact in Sending Areas; DUNCAN, Kenneth, RUTLEDGE, lan (comps.). La tierra y la mano de obra en América Latina. Ensayo sobre el desarrollo del capitalismo agrario en los siglos XIX y XX; GORDILLO, Gastón. Después de los ingenios: la mecanización de la zafra saltojujeña y sus efectos sobre los indígenas del Chaco centro-occidental; GRECO, María Gabriela. Alternativas laborales y movilidad espacial de la población: los cambios en la demanda de mano de obra por parte de la agroindustria azucarera y sus efectos socioespaciales; SASSONE, Susana. Breve geografía histórica de la migración boliviana en la Argentina. 
de los flujos "renunciando al enfoque exclusivamente focalizado sobre el espacio de llegada o el de origen"15. En este sentido, se considera que los mercados de trabajo agrario se vincularon con un "espacio multi-polarizado, estructurado por desplazamientos alternantes ${ }^{\prime 16}$ y configurado por los procesos de reestructuración productiva.

En la actualidad, algunos estudios realizados en Argentina muestran las modalidades a través de las cuales los complejos agroindustriales organizan la circulación de mano de obra, analizan las condiciones de trabajo de los migrantes, y señalan que, en los últimos años, la legislación laboral tuvo como objetivo avanzar en los controles de informalidad y seguridad laboral ${ }^{17}$. Por otra parte, frente a las especificidades del mercado laboral agrario y los cambios que lo afectaron recientemente, ciertas investigaciones han advertido acerca de los alcances y las limitaciones de las fuentes de información disponibles que dificultan el estudio de la complejidad y heterogeneidad del/losmercado(s) de trabajo agrario y su(s) dinámica(s) ${ }^{18}$ de movilidad creciente.

Aún frente a las limitaciones de los datos estadísticos, las investigaciones de algunos sociólogos evidencian la importancia de los trabajadores temporarios migrantes en estos mercados laborales, puesto que su cantidad es significativa y sus condiciones de trabajo son sumamente precarias. Se estudia la existencia de diversas "modalidades de contratación de trabajadores provistos por empresas de servicios, por agentes más o menos informales (o 'cuadrilleros') o por distintas figuras que operan al amparo de la ambigüedad de la legislación respectiva"19.

${ }^{15}$ CORTES, Genevieve. Migraciones, construcciones transnacionales y prácticas de circulación: un enfoque desde el territorio, p. 39.

16 Ibidem.

17 BENENCIA, APARICIO, op. cit.; MASTRANGELO, Andrea, TRPIN, Verónica. Trabajo rural en producciones que Argentina exporta: una síntesis de características relevadas en estudios etnográficos recientes; PIZARRO, Cynthia. Sanidad, calidad: bioregulación y disciplinamiento. Las buenas prácticas agrícolas en la producción hortícola argentina; PIZARRO, Cynthia, TRPIN, Verónica. Trabajadores frutícolas y hortícolas en la Argentina: una aproximación socioantropológica a las prácticas de reproducción y de resistencia de las condiciones laborales.

${ }^{18}$ QUARANTA, Germán. Estructura ocupacional, características de la demanda y perfil de la oferta laboral en el agro argentino a principios de la década actual, señala que los censos de población resultan útiles para caracterizar la oferta de empleo y captar mejor la existencia de relaciones salariales (registradas o no), su magnitud, composición y distribución regional, pero no logran captar adecuadamente el trabajo transitorio en el sector agropecuario de aquellos trabajadores que a la fecha de realización del censo se encuentran inactivos, desocupados u ocupados en otra actividad (por el período de referencia que se utiliza para su registro). Los censos agropecuarios, por su parte, resultan útiles para describir la demanda de trabajo (cantidad y tipo de trabajadores empleados, tipos de establecimientos, cantidad de jornales contratados según tarea realizada), aunque tradicionalmente se ha constatado el subregistro de trabajadores permanentes. Esto se debe a la complejidad de la información sobre la movilidad territorial de los trabajadores y a las dificultades que implica su registro. A esto se suman las diferencias entre los cuestionarios censales, lo cual dificulta en muchas ocasiones el análisis comparativo intercensal.

19 NEIMAN, Guillermo. Los estudios sobre el trabajo agrario en la última década: una revisión para el caso argentino, p. 5, comillas en el original. 
Dicho autor advierte que, aunque la intermediación y la tercerización no son equiparables, ambas cumplen un rol central en el acceso de mano de obra en empresas.

La intermediación laboral ha sido abordada como mecanismo de reclutamiento, transporte y disciplinamiento de trabajadores, prestando especial atención a los diferentes sujetos involucrados, como por ejemplo empresas de recursos humanos, cooperativas, enganchadores y transportistas. Las investigaciones coinciden en observar que la expansión de la intermediación laboral en producciones pampeanas y extrapampeanas acrecienta la precarización de las condiciones laborales, plantean importantes dificultades para la intervención del estado con controles efectivos y limitan las posibilidades de sindicalización ${ }^{20}$.

En este contexto de creciente fragmentación social en los espacios agrarios y atendiendo a la diversidad de orígenes nacionales y regionales de la mano de obra que circulan a través de las fronteras o dentro del territorio argentino, la movilidad de trabajadores está también atravesada por sus pertenencias étnicas y marcaciones racializantes. En esta línea, el diálogo entre la antropología, la sociología y la geografía es central para estudiar las migraciones estacionales, las migraciones limítrofes y la inserción en nichos laborales y productivos no ocupados por "nativos" 21 .

Dichos cruces se observan en investigaciones sobre las experiencias de migrantes procedentes de Bolivia y de las provincias del noroeste argentino en la producción hortícola en diferentes puntos de la Argentina. Estos estudios analizan las trayectorias laborales y migratorias así como las modalidades que presenta la movilidad social ascendente de estos migrantes en una producción específica ${ }^{22}$.

${ }^{20}$ AGUILERA, María Eugenia. Modalidades de intermediación en la contratación de cosecheros citrícolas en Tucumán; APARICIO, Susana, BERENGUER, Paula, RAU, Víctor. Modalidades de intermediación en los mercados de trabajo rurales en Argentina; BENENCIA, APARICIO, op. cit.; QUARANTA, Germán, FABIO, Francisco. Intermediación laboral y mercados de trabajo en agriculturas reestructuradas: el caso del Valle de Uco, Mendoza, Argentina; STEIMBREGER, Norma, TRPIN, Verónica, BENDINI, Mónica. Intermediación laboral en el acceso y gestión del trabajo estacional en la fruticultura rionegrina.

${ }^{21}$ BENDINI, Mónica, RADONICH, Martha, STEIMBREGER, Norma. Nuevos espacios agrícolas, mercado de trabajo y migraciones estacionales; CIARALLO, Ana. Tensiones, resistencias y desigualdades en los nuevos escenarios de la horticultura en el norte de la Patagonia argentina; GIARRACCA, Norma, BIDASECA, Karina, MARIOTTI, Daniela. Tucumanos y tucumanas: zafra, trabajo, migraciones e identidad; KARASIK, Gabriela. Migraciones, trabajo y corporeidad. Bolivianos y nativos en el trabajo rural y el servicio doméstico de Jujuy; PIZARRO, Cynthia (coord.). Migraciones internacionales contemporáneas: estudios para el debate; RADONICH, CIARALLO, TRPIN, op. cit.; TRPIN, Verónica. Aprender a chilenos: identidad, trabajo y residencia de familias migrantes en el Alto Valle de Río Negro.

22 BENENCIA, Roberto. Bolivianización de la horticultura en la Argentina: procesos de migración transnacional y construcción de territorios productivos; BENENCIA, Roberto, QUARANTA, Germán, SOUZA CASADINHO, Javier (coords.). Cinturón hortícola de la Ciudad de Buenos Aires: cambios sociales y productivos; BENENCIA, Roberto, RAMOS, Diego. Revisitando el cinturón hortícola de Río Cuarto, Córdoba: afianzamiento y cambios en el fenómeno migratorio de familias bolivianas; CIARALLO, op. cit.; GARCíA, Matías. Uso y acceso a la tierra en el marco 


\section{Etnicización y racialización de la mano de obra migrante}

A lo largo de la historia argentina, diversos grupos de migrantes internos e internacionales han contribuido de manera significativa en la producción de bienes y servicios, participando en la construcción de novedosos territorios socioculturales. Sin embargo, han sido catalogados por los discursos gubernamentales, mediáticos y del sentido común como "indeseables", siendo objeto en distintos momentos de discriminaciones xenófobas ${ }^{23}$. A lo largo del siglo XX y de lo que va del XXI, chilenos, paraguayos y bolivianos, conjuntamente con personas pertenecientes a distintos pueblos originarios $y / o$ a sectores pauperizados de algunas provincias argentinas, se han ubicado en los escalones más bajos de las jerarquías laborales en áreas rurales, configurando mercados de trabajo segmentados étnicamente. Por otra parte, aun cuando algunos/as migrantes hayan logrado cierta movilidad socio-económica, se les han asignado posiciones etnicizadas y racializadas en las estructuras de clasificación de la otredad.

Dentro de estos grupos móviles, quienes atraviesan las fronteras internacionales son definidos como migrantes laborales. Sin embargo, algunos investigadores señalan que las categorías inmigrante-emigrante son construcciones que no comprenden a todos/as los/las que atraviesan los límites entre los estados nacionales, puesto que el poder coercitivo, controlador y clasificador del Estado asigna dichos status sólo a aquellos a quienes se les adscribe una motivación económica para buscar trabajo en otro país, diferenciándolos/as de los/as exiliados/as, estudiantes, turistas y expatriados/as, entre otros/as ${ }^{24}$.

Tal como lo planteó Sayad ${ }^{25}$, los movimientos territoriales internacionales son alentados por la expansión económica del capitalismo y la condición de inmigrantes recae sobre los/as trabajadores/as provisorios/as, temporarios/as, en tránsito, cuya estadía autorizada depende de que tengan un empleo. En el caso de los migrantes internos, el hecho de que sean tolerados por los "nativos" también depende de que se considere que realicen algún "aporte" a la sociedad a través de su trabajo. Se produce entonces una forma particular de segmentación del mercado laboral que asigna a ciertos trabajadores a las posiciones laborales

del muevo modelo productivo de la horticultura platense; RIVERO SIERRA, op. cit.; PIZARRO, Cynthia (ed.). "Ser boliviano" en la región metropolitana de la ciudad de Córdoba. Localización socio-espacial, mercado de trabajo y relaciones interculturales; PIZARRO, Cynthia. Partir y volver entre Argentina y Bolivia: trayectorias migratorias transnacionales intersección de desigualdades; PIZARRO, Cynthia (ed.). Bolivianos y bolivianas en la vida cotidiana cordobesa: Trabajo, derechos e identidad en contextos migratorios, PIZARRO, Cynthia (ed.). Bolivian Labor Immigrants ` Experiences in Argentina.

${ }^{23}$ BELVEDERE, CAGGIANO, CASARAVILLA et alii, op. cit.

${ }^{24}$ PEDREÑO CÁNOVAS, Andrés. Sociedades etnofragmentadas; PIZARRO, Cynthia. Clasificar a los otros migrantes: las políticas de migración argentinas como productoras de etnicidad y desigualdad.

${ }^{25}$ SAYAD, Abdelmalek. A imigração ou os paradoxos de alteridade. 
más precarias y vulnerables, por el hecho de que compartirían un conjunto de características supuestamente innatas debido a su etnicidad ${ }^{26}$.

Fenton ${ }^{27}$ plantea que las clasificaciones e identidades étnicas han operado como una forma de control sobre la economía y la sociedad en distintos momentos históricos tales como la esclavitud, la dominación colonial y el desarrollo del capitalismo y de los estados-nación. Relaciona dichas situaciones históricas con distintos tipos de grupos étnicos: los migrantes internacionales que se desempeñan como trabajadores y/o comerciantes en las sociedades de destino, los pueblos subsumidos en o entre diferentes estados-nación, las minorías aborígenes y los afrodescendientes.

De manera pionera, estudios realizados por antropólogos críticos tales como Melliasoux ${ }^{28}$ y Wolf ${ }^{29}$ han destacado que la relación desigual entre capital y trabajo es favorecida, entre otros factores, por la etnicización de las relaciones de producción, y han señalado que constituye una condición necesaria del sistema capitalista. Más recientemente, Morberg ${ }^{30}$ y Holmes $^{31}$ especificaron la operatoria de estos procesos en contextos migratorios en Estados Unidos. En Argentina, los estudios de algunos antropólogos y otros cientistas sociales retoman esta línea de análisis para dar cuenta de las características de la discriminación étnico-nacional de los migrantes laborales internacionales ${ }^{32}$.

${ }^{26}$ JIMÉNEZ ZUNINO, Cecilia. Desclasamiento y reconversiones en las trayectorias de los migrantes argentinos de clases medias; PIZARRO, Cynthia. Discurso racializante y segmentación étnico-nacional del mercado laboral: Trabajadores bolivianos en un cortadero de ladrillos de Córdoba, Argentina.

27 FENTON, Steve. Ethnicity: Racism, Class and Culture.

${ }^{28}$ MELLIASOUX, Claude. Mujeres, graneros y capitales.

${ }^{29}$ WOLF, Eric. Europa y la gente sin historia.

${ }^{30}$ MORBERG, Mark. Myth of Ethnicity and Nation: Immigration, Work and Identity in the Belize Banana Industry.

${ }^{31}$ HOLMES, Seth. "Oaxacans Like to Work Bent Over": The Naturalization of Social Suffering among Berry Farm Workers.

32 ATAIDE, Soraya. Incorporación laboral de inmigrantes bolivianos en la agricultura salteña. Estudio de caso en el municipio de Apolinario Saravia; BAEZA, Brígida. Trabajadores de la construcción: bolivianos y paraguayos en Comodoro Rivadavia. Identificaciones, diferenciaciones y conflictos; BRUNO, Sebastián. Inserción laboral de los migrantes paraguayos en Buenos Aires. Una revisión de categorías: desde el "nicho laboral" a la "plusvalía étnica"; COURTIS, Corina, PACECCA, María Inés. Género y trayectoria migratoria: mujeres migrantes y trabajo doméstico en el Área Metropolitana de Buenos Aires; HALPERN, Gerardo. Etnicidad, inmigración y política: representaciones y cultura política de exiliados paraguayos en Argentina; KARASIK, op. cit.; MAGLIANO, María José. Los significados de vivir "múltiples presencias": Mujeres bolivianas en Argentina; MARGULIS, Mario. La racialización de las relaciones de clase; PIZARRO, Cynthia. Trabajadores paraguayos en la producción forestal del Delta Inferior del río Paraná; PIZARRO, Cynthia, FABBRO, Pablo, FERREIRO, Mariana. "No conozco trabajo más perro que éste": Reproducción y resistencia de la subalternidad de los trabajadores bolivianos en un cortadero de ladrillos de la ciudad de Córdoba, Argentina; PIZARRO, Cynthia, MORENO, Marta Silvia. Differential Migration Pathways of Bolivian Women Working in Horticultural Fields in Mendoza; PÉREZ, Natalia Evangelina. "Quiero que mis hijos estudien, para que no trabajen a pico y pala como nosotros": prácticas escolares de marcación y (des)marcación de la etnicidad boliviana. 
Por otra parte, Ratier ${ }^{33}$ destacó de manera pionera la asignación de lugares de identidad etnicizados para los trabajadores que comenzaron a circular entre el campo y la ciudad a mediados del siglo XX: los "cabecita negras"; y otros investigadores ${ }^{34}$ han hecho lo mismo para los trabajadores estacionales pertenecientes a pueblos originarios.

Pizarro $^{35}$ ha discutido sobre la pertinencia de emplear el concepto de etnicidad para referir a aquellos procesos de marcaciones identitarias que justifican y legitiman la asignación de ciertos migrantes internacionales en las jerarquías laborales más precarizadas. La autora remarca los aportes de diversos antropólogos ${ }^{36}$ que señalaron que, aun cuando las clasificaciones de etnicidad se estructuran en base a diferencias culturales o raciales entre la mismidad y la otredad, no plasman características innatas o primordiales de los grupos humanos. Por el contrario, estas marcaciones son producidas en contextos históricos particulares. La auto y hetero-asignación de etnicidad da forma a un ordenamiento social jerárquico que legitima la dominación de unos frecuentemente concebidos como no-étnicos y la subordinación de otros que son marcados como tales, quienes vivencian dichas marcaciones y muchas veces actúan en nombre de ellas.

Este proceso tiene lugar a través de los mecanismos de etnicización y racialización que marcan la alteridad apelando a justificativos que señalan la diferencia cultural o biológica respectivamente. Si bien estas categorías son distintas, generalmente operan entrelazadas al esencializar las diferencias culturales. Ambos procesos son logrados a través de ciertas nominaciones hegemónicas que justifican y naturalizan la desigualdad, la que es explicada a través de conexiones pseudo-causales que conectan a ciertas minorías, consideradas como comunidades de descendencia, con determinados rasgos biológicos (genéticos y fenotípicos), psicológicos, sociales y culturales colectivos, que son considerados como invariables.

Es decir, dan forma a una política cultural a través de la cual se reproducen o transforman los sistemas clasificatorios de la otredad y las desigualdades que los mismos habilitan. Estos procesos pueden ser llevados a cabo desde arriba, en el marco de políticas de identidad a través de las cuales los sectores hegemónicos moldean las identidades colectivas, fijando y naturalizando los

\footnotetext{
${ }^{33}$ RATIER, Hugo. El cabecita negra.

${ }^{34}$ GORDILLO, op. cit.; TRINCHERO, Hugo. Los dominios del demonio. Civilización y barbarie en las fronteras de la nación. El chaco central.

${ }^{35}$ PIZARRO, Cynthia. La bolivianidad en disputa. (Des)marcaciones de etnicidad en contextos migratorios.

36 Tales como BRIONES, Claudia. La alteridad del "Cuarto Mundo": una deconstrucción antropológica de la diferencia; BRIONES, Claudia. Cartografías argentinas: políticas indigenistas y formaciones provinciales de alteridad; COMAROFF, Jean, COMAROFF, John. Home-made Hegemony: Modernity, Domesticity, and Colonialism in South Africa; FENTON, op. cit.; y GRIMSON, Alejandro. Los límites de la cultura: crítica de las teorías de la identidad.
} 
marcos interpretativos. Esto es cumplimentado por los grupos hegemónicos a través de diversas modalidades de discriminación ${ }^{37}$. Una de ellas consiste en enfatizar las diferencias de manera tal de inferiorizar a los subalternos con el objeto de legitimar y proteger la propia superioridad. Otra forma consiste en jerarquizar a los grupos etnicizados, fomentando su integración desigual en los escalones más bajos de la jerarquía social.

Al mismo tiempo, las políticas identitarias de los sectores subalternos alterizados resignifican las marcaciones hegemónicas tanto para confrontarlas como para adecuarse a los lugares de identificación asignados. Por ejemplo, la invisibilización de dicha diferencia puede tener distintos sentidos que van desde el polo del silencio como forma de resistencia hasta la negación como apuesta para lograr el pasaje a través de las fronteras étnicas.

En síntesis, la exaltación o invisibilización de determinadas características culturales o bio-psicológicas puede naturalizar o cuestionar -a través de estrategias de re/des/etnicización tales como el proteccionismo, la inferiorización, el distanciamiento, la negación y/o el refuerzo de las diferencias culturales-raciales-, la designación estereotipada y jerárquica de los grupos auto o heteroetnicizados a ciertos nichos dentro de la división social del trabajo. De este modo, en el marco de una economía política de la cultura, las diferencias de clase son interpretadas como diferencias culturales, dando lugar a la etnicización de las relaciones de producción.

\section{Migraciones, trabajo y género}

Tal como hemos planteado más arriba, hasta años recientes, la clase constituyó un concepto dominante para analizar las relaciones laborales en los mercados de trabajo en los que participan migrantes. Así, distintas investigaciones se limitaron a analizar las relaciones sociales basadas en relaciones productivas o económicas. El estudio de la intersección de las desigualdades de clase con las étnico-nacionales y raciales se incorporó gradualmente. Es sólo en los últimos años que se ha reparado en la incidencia de las desigualdades de género.

A instancias de ciertos desarrollos conceptuales novedosos, los/as investigadores/as de las migraciones laborales debimos asumir el desafío de librarnos tanto del determinismo económico como del etnocentrismo y nacionalismo metodológico ${ }^{38}$. Asimismo, fuimos interpelados/as por los mismos procesos que estudiamos, que nos incitaron a despojarnos del androcentrismo ${ }^{39}$ y de un esquema binario en el abordaje de la división del trabajo.

\footnotetext{
37 WIEVIORKA, Michel. Diferencias culturales, racismo y democracia.

${ }^{38}$ BASCH, Linda, GLICK SCHILLER, Nina, SZANTON BLANC, Cristina. Nations Unbound. Transnacional Projects, Postcolonial Predicaments and Deterritorialized Nation-States; SILVERSTEIN, Paul. Immigrant Racialization and the New Savage Slot: Race, Migration, and Immigration in the New Europe.

${ }^{39}$ MAGLIANO, María José. El género y la historia oral en los estudios sobre las migraciones
} 
El análisis de las relaciones laborales asimilando lo productivo a lo masculino ha reproducido las concepciones dominantes "sobre la diferenciación sexual del trabajo y la división tradicional de roles: varones productivos (y activos) y mujeres reproductivas (dependientes)" ${ }^{\prime \prime 0}$. Los estudios feministas advirtieron sobre la reproducción de dicho binarismo y sobre las limitaciones que supone considerar a las migraciones como exclusivamente laborales/masculinas sin contemplar la participación de las mujeres en este tipo de movilidad territorial. En esa dirección, diversas investigadoras plantean que las motivaciones, trayectorias y proyectos migratorios de varones y mujeres presentan características diferenciales según los patrones que asuman las relaciones patriarcales tanto en las sociedades de origen como en los nodos de los territorios circulatorios de los y las trabajadores/as migrantes ${ }^{41}$.

Por otra parte, la diferencia salarial, la segregación de los empleos, el papel de los estereotipos de género en la definición de las calificaciones y ocupaciones, la escasa o nula representación de las mujeres en organizaciones gremiales, son algunos elementos que dan cuenta del modo en que el género opera más allá del ámbito privado ${ }^{42}$. Algunos estudios plantean que ciertas calificaciones laborales se construyen sobre la base de clasificaciones genéricas que sólo se toman en cuenta las cualidades asociadas a lo masculino -como la mayor capacidad de fuerza física-, mientras que las cualidades asociadas a lo femenino tales como la paciencia o la delicadeza, son consideradas destrezas o atributos "naturales"43.

internacionales: aportes y desafíos, plantea que la incorporación de las perspectivas de género tuvo dos aspectos sumamente significativos, dos momentos: uno de deconstrucción y otro de reconstrucción del conocimiento. El primero consiste en la desconstrucción del conocimiento occidental androcéntrico que permitió rescatar a actores/as que habían estado silenciados y también reconocer el papel de lo femenino, de lo doméstico y lo cotidiano en los procesos de transformación histórica. Por otro lado, se da una reconstrucción del conocimiento en las ciencias sociales permitido por la incorporación de parámetros sensibles al género, lo que lleva a generar propuestas epistemológicas que ponen en tensión el paradigma positivista, objetivo, racional y neutral que había caracterizado a los estudios sociales.

${ }^{40}$ MALLIMACI BARRAL, Ana. Nuevas miradas. Aportes de la perspectiva de género al estudio de los fenómenos migratorios, p. 120.

${ }^{41}$ BASTIA, Tania. 'I Am Going, with or without You': Autonomy in Bolivian Transnational Migrations; COURTIS, PACECCA, op. cit.; PEDONE, Claudia. Varones aventureros vs. madres que abandonan: reconstrucción de las relaciones familiares a partir de la migración ecuatoriana; PIZARRO, Cynthia. Intersection of Inequalities: Migratory Trajectories, Labor Experiences, and Family Life of Bolivian Women on the Outskirts of Buenos Aires and Córdoba; PIZARRO, Cynthia, BASUALDO, María Lourdes, FERREIRO, Mariana. Practices of Resistance among Young Bolivian Immigrants Working in a Brick Factory in Córdoba; PIZARRO, MORENO, op. cit.; ROSAS, Carolina. Dilemmas and Decisions of Peruvian Couples before Migrating to Argentina.

${ }^{42} \mathrm{RADONICH}$, Martha, TRPIN, Verónica. Mujeres migrantes en la organización de territorios rurales en el Alto Valle de Río Negro.

${ }^{43}$ BENDINI, Mónica, PESCIO, Cristina. Mujer y trabajo: las empacadoras de fruta del Alto Valle; MIRALLES, Glenda, RADONICH, Martha. De trabajadoras familiares y asalariadas de los Valles de los ríos Negro y Neuquén. 
Asimismo, se ha demostrado que los sentidos que varones y mujeres le otorgan al trabajo son heterogéneos, así como las condiciones y relaciones laborales en las que los/las migrantes se ven involucrados/as. Mariela Pena incita a considerar "las experiencias de las mujeres" en plural, señalando que no existe la 'mujer universal' como contrapartida del imaginario 'hombre universal', sino también a dar cuenta de la visibilización de lo masculino y lo femenino como categorías construidas e imbricadas con otras también culturales como la raza y la clase socia ${ }^{44}$. En esta dirección, Pizarro ${ }^{45}$ señala que la mirada optimista que postula que las migraciones de las mujeres suponen de por sí una mayor autonomía o empoderamiento, debe ser matizada teniendo en cuenta la manera en que se intersectan las desigualdades de género con las de clase, etnia, procedencia regional y generación.

Las escasas investigaciones que abordan las migraciones y el trabajo rural protagonizados por mujeres, dan cuenta de cómo se encuentran arraigados modos androcéntricos de pensar la clase. En la Argentina, constituyen una referencia los trabajos de Vazquez Laba ${ }^{46}$ sobre mujeres insertas en la producción tabacalera en la provincia de Jujuy y la citrícola en Tucumán, mientras que Pizarro analiza el trabajo de las mujeres en la producción hortícola en Córdoba y en Buenos Aires, así como el trabajo de los varones en la producción forestal en el Delta Inferior del río Paraná ${ }^{47}$.

Por otra parte, se han realizado investigaciones sobre el empleo de mujeres temporarias en la clasificación y empaque de fruta y su presencia en la organización sindical; sobre migrantes chilenas en las chacras frutícolas ${ }^{48} ;$ y sobre migrantes bolivianas en la horticultura del Valle Medio de Río $\mathrm{Negro}^{49}$, en la producción de ajo en Mendoza ${ }^{50}$ y en la fabricación de ladrillos en Córdoba ${ }^{51}$.

Las investigaciones mencionadas anteriormente comparten el trabajo de campo intensivo y la realización de entrevistas y observaciones con el objetivo de describir y analizar las diversas experiencias de mujeres migrantes y trabajadoras. Dichos estudios coinciden en considerar la "interseccionalidad"

${ }^{44}$ PENA, Mariela. Aportes de la incorporación de perspectivas feministas a las investigaciones con técnicas orales, p. 10, comillas en el original.

${ }^{45}$ PIZARRO, Intersection of Inequalities..., op. cit.

${ }^{46}$ VÁZQUEZ LABA, Vanesa. Re-pensando la división sexual del trabajo familiar: aspectos teóricos y empíricos para la interpretación de los modelos de familia en el noroeste argentino.

47 PIZARRO, Intersection of Inequalities..., op. cit.; PIZARRO, Trabajadores paraguayos..., op. cit.

${ }^{48}$ BENDINI, Mónica, BONACCORSI, Nélida (comps.). Con las puras manos: mujer y trabajo en regiones frutícolas de exportación; MIRALLES, Glenda. Entre la casa y el galpón ihay lugar para el sindicato?; RADONICH, TRPIN, op. cit.

${ }^{49}$ CIARALLO, Ana, TRPIN, Verónica. Migration and Labor Market in Horticulture: Bolivian Families in the Middle Valley of the Río Negro, Argentine Patagonia; TRPIN, Verónica, BROUCHOUD, Silvia. Mujeres migrantes en producciones agrarias de Río Negro: aportes para abordar la interseccionalidad en las desigualdades.

${ }^{50}$ PIZARRO, MORENO, op. cit.

51 PIZARRO, BASUALDO, FERREIRO, op. cit. 
como un "sistema complejo de estructuras de opresión que son múltiples y simultáneas" ${ }^{\prime 52}$. Este abordaje contribuye a visualizar de qué manera convergen distintos tipos de discriminación ${ }^{53}$, insistiendo en que "las estructuras de clase, racismo, género y sexualidad no pueden tratarse como 'variables independientes' porque la opresión de cada una está inscrita en las otras -es constituida por y es constitutiva de la otras-" ${ }^{54}$.

Para Gil Hernández ${ }^{55}$, la interseccionalidad es un modelo de análisis de las diferencias sociales que permite comprender las relaciones de poder determinadas por posiciones que se cruzan y encuentran, como en el caso de quien es indígena, mujer y lesbiana. Las relaciones que se establecen entre diferentes categorías como clase, raza, género, sexualidad, entre otras, no son siempre las mismas. Algunos/as investigadores/as sostienen que se trata de una múltiple discriminación cuyo efecto es multiplicador puesto quela yuxtaposición de las diferentes discriminaciones posiciona al/ a la sujeto/a en una situación de mayor desigualdad ${ }^{56}$. Por otro lado, otros/as plantean que la sexualidad, género o sexo deben ser abordadas como metáforas raciales, donde la raza se construye como base en la diferencia sexual.

\section{Reflexiones finales: repensando las desigualdades en las circulaciones territoriales en contextos rurales de los/las trabajadores/as migrantes}

En las últimas décadas, los estudios migratorios sobre espacios rurales en la Argentina involucraron cruces interdisciplinares desde la antropología, la sociología y la geografía, problematizando categorías como territorio, fronteras, circulación y movilidad, y combinando diversos enfoques teóricometodológicos. El énfasis territorial vinculado a los aportes de la geografía requiere atender la dimensión temporal como modo de conocer las trayectorias de los y las migrantes y las históricas tensiones entre las construcciones territoriales protagonizadas por estos/as sujetos/as y las configuraciones organizadas por el Estado y el capital. En diálogo con estas perspectivas los aportes antropológicos han sido centrales en la descripción de los procesos de etnicización y racialización de colectivos de modo de analizar cómo el énfasis en las diferencias inferioriza a los subalternos con el objeto de legitimar y proteger la propia superioridad de determinadas clases sociales sobre otras.

52 CRENSHAW, op. cit., p. 359.

53 ANTHIAS, op. cit.

${ }^{54}$ BRAH, Avtar. Diferencia, diversidad, diferenciación, p. 138, comillas en el original.

${ }^{55}$ GIL HERNÁNDEZ, Franklin. Estado y procesos políticos. Sexualidad e interseccionalidad.

${ }^{56}$ MALLIMACI BARRAL, Ana. Localizando el sentido de las desigualdades. Inclusiones y exclusiones de los/as bolivianos/as en Ushuaia retoma las metáforas multiplicadoras que critican a las aditivas (siguiendo a PARELLA RUBIO, Sonia. El trasvase de desigualdades de clase y etnia entre mujeres de servicios de proximidad) y argumenta que las desigualdades de clase, etnia-raza y género se multiplican puesto que no es posible experimentar el género sin simultáneamente experimentar la raza y la clase. 
Por otra parte, las discusiones sobre la incidencia de las desigualdades de género en las condiciones laborales de los y las migrantes también han atravesado los estudios sobre la movilidad territorial de los/as trabajadores/ as rurales. Sin embargo, la dimensión referida a las desigualdades basadas en las diferencias entre generaciones-cohortes aún no ha sido analizada por las investigaciones sobre migraciones en espacios rurales, y ha sido incorporada tímidamente en los estudios sobre movilidades en otras áreas.

Consideramos que es necesario destacar los aportes que brinda la categoría interseccionalidad a los estudios migratorios, puesto que incita a indagar sobre las maneras en que se yuxtaponen las desigualdades de clase, género, etnia-raza y generación en las trayectorias y experiencias migratorias. Esto implica aceptar que la posición de clase no es el único condicionamiento que atraviesa la articulación de los y las trabajadores/as migrantes en los mercados laborales en contextos rurales. Por el contrario, permite problematizar la manera en que dichas yuxtaposiciones coadyuvan a que ciertos/as trabajadores/as ocupen las posiciones más bajas en las jerarquías laborales. Asimismo, el análisis de la interseccionalidad abre intersticios para observar procesos de movilidad ascendente dentro de trayectorias individuales que tensionan las marcaciones étnicas y raciales y su relación con los "destinos de clase".

En esa dirección, diversos/as antropólogos/as han remarcado desde hace décadas la importancia de estudiar las desigualdades de clase en las investigaciones sobre sobre género, etnicidad y raza, pero teniendo la precaución, según Fonseca ${ }^{57}$, de no definir los objetos de estudio exclusivamente en función de las relaciones de producción. Por su parte, $\operatorname{Ortne}^{58}$ destaca la necesidad de indagar sobre el problema del poder, la dominación y la autoridad para poner en evidencia las vinculaciones entre la cultura popular -en la que las personas definen sus identidades, sus límites, su espacio- con el orden establecido, y las relaciones complejas y problemáticas entre las distinciones de clase y género, etnia y raza.

Incluso la llamada "teoría de las vinculaciones mutuas", se preocupó desde los 1970s por vincular raza, clase y género como sistemas combinados que definen identidades y relaciones de desigualdad enmarcados históricamente. Las tres categorías señaladas no se conciben como categorías per se, sino como punto de partida para rastrear "la mutabilidad de las estructuras ideológicas de dominación que se construyen a partir de estas distinciones" ${ }^{\prime \prime 2}$. En esta línea, Quijano ${ }^{60}$ señala que el capitalismo mundial, colonial/moderno, es una estructura de poder que articula todas las formas históricas de trabajo, control

\footnotetext{
${ }^{57}$ FONSECA, Claudia. Familia, fofoca e honra. Etnografia de relações de gênero e violência em grupos populares; FONSECA, Claudia. La clase social y su recusación etnográfica.

${ }^{58}$ ORTNER, Sherry. La teoría antropológica desde los años sesenta.

${ }^{59}$ BRIONES, op. cit., p. 41.

${ }^{60}$ QUIJANO, Aníbal. Colonialidad del poder, eurocentrismo y América Latina.
} 
y explotación, en el marco de las cuales los grupos sociales se clasifican y son clasificados según tres líneas: trabajo, raza, género.

Falquet ${ }^{61}$ recomienda abordar las relaciones de género, clase y género no tanto como interseccionadas sino como "consustanciales" y "co-formadas" a partir de un objeto concreto: la reorganización de la división del trabajo y en especial del "trabajo considerado como femenino" en la actual dinámica del capitalismo que modifica simultáneamente las relaciones sociales de sexo, de raza y de clase en contextos que portan históricas marcas coloniales. Observa que la mayor parte del "trabajo desvalorizado" ha sido ejecutado por la población migrante. Señala que la perspectiva de la "co-formación" de las relaciones de poder explica por qué esos trabajos -si bien pueden ser ejercidos por individuos de sexo masculino, en especial si han sido etnicizados y naturalizados con este fin (migrantes, esclavos o colonizados)-, son resueltos en su mayor proporción por personas socialmente construidas como mujeres etnicizadas y racializadas.

De este modo, consideramos que es un desafío analizar cómo la propia condición de migrante constituye un factor que se agrega a la intersección de desigualdades, debido a que las estructuras de opresión son múltiples y simultáneas y a que las posiciones de las personas que circulan territorialmente son heterogéneas. En esta dirección, Pizarro ${ }^{62}$ señala que las diferencias al interior de grupos migratorios provenientes del mismo estado-nación y las experiencias diferenciales de trabajadoras migrantes originarias de sectores campesinoindígenas de un mismo país deben ser recuperadas como parte de los análisis.

Finalmente, queremos resaltar la capacidad de agencia de los y las trabajadores/as migrantes. En los últimos años, junto con otras investigadoras, hemos comenzado a poner en evidencia que, en Argentina, las formas específicas de subordinación y de poder son experimentadas por los/las migrantes de diversas maneras, de acuerdo a sus trayectorias sociales y a los condicionamientos estructurales diferenciales en cada contexto temporalespacial; y hemos señalado que estos procesos habilitan, a la vez, variadas formas de resistencia ${ }^{63}$.

Tal como hemos visto en este artículo, tanto los factores macro como los meso y micro estructurales moldean las circulaciones territoriales contemporáneas de manera tal que las personas clasificadas como inmigrantes suelen articularse en ciertos mercados de trabajo segmentados por desigualdades de clase, etnia y género. La intersección de dichas situaciones de

\footnotetext{
${ }^{61}$ FALQUET, op. cit.

${ }^{62}$ PIZARRO, op. cit.

63 PIZARRO, op. cit.; PIZARRO, BASUALDO, FERREIRO, op. cit.; PIZARRO, TRPIN, op. cit.; RADONICH, TRPIN, op. cit.
} 
opresión -presentes y pasadas- define sus posiciones posibles en el territorio conformado por sus trayectorias sociales y circulatorias. Consideramos que dar cuenta de estas cuestiones es un desafío para el estudio de las migraciones laborales en territorios rurales. A la vez, restan estudiar las yuxtaposiciones de otras desigualdades, tales como las de generación, status migratorio jurídicoadministrativo (migrante regular, irregular, etc.), región de procedencia (áreas indígeno-campesinas, áreas urbanas periféricas, etc.) y nodos por los que se circuló previamente. Asimismo, quedan pendientes análisis de este tipo en otros ámbitos laborales. Finalmente, otra línea de investigación que amerita ser profundizada se refiere a las maneras en que los/las migrantes recrean, resisten y/o confrontan las posiciones sociales que les son asignadas.

\section{Bibliografía}

AGUILERA, María Eugenia. Modalidades de intermediación en la contratación de cosecheros citrícolas en Tucumán. In APARICIO, Susana; BENENCIA, Roberto (coords.). Antiguos y nuevos asalariados en el agro argentino. Buenos Aires: La Colmena, 2001, p. 15-28.

ALBANESI, Roxana; PREDA, Graciela. El enfoque territorial como propuesta de intervención para el desarrollo. Reflexiones desde una perspectiva latinoamericana. Ponencia presentada en Encuentro sobre Enseñanza del Desarrollo Rural en América Latina. Bogotá: Universidad Javeriana, 2005.

ANTHIAS, Floya. The Material and the Symbolic in Theorizing Stratification: Issues of Gender, Ethnicity and Class. British Journal of Sociology, v. 52, n. 3, 2001, p. 367-390.

APARICIO, Susana; BERENGUER, Paula; RAU, Víctor. Modalidades de intermediación en los mercados de trabajo rurales en Argentina. Cuadernos de Desarrollo Rural, v. 1, n. 53, 2004, p. 59-79.

ATAIDE, Soraya. Incorporación laboral de inmigrantes bolivianos en la agricultura salteña. Estudio de caso en el municipio de Apolinario Saravia. Ponencia presentada en el V Congreso Argentino y Latinoamericano de Antropología Rural, 2013.

BAEZA, Brígida. Trabajadores de la construcción: bolivianos y paraguayos en Comodoro Rivadavia. Identificaciones, diferenciaciones y conflictos. In KARASIK, Gabriela (coord.). Migraciones internacionales. Reflexiones y estudios sobre la movilidad territorial contemporánea. Buenos Aires: CICCUS, 2013, p. 361-382.

BALAN, Jorge. Migraciones temporarias y mercado de trabajo rural en América Latina. Buenos Aires: CEDES, 1980.

BASCH, Linda; GLICK SCHILLER, Nina; SZANTON BLANC, Cristina. Nations Unbound. Transnacional Projects, Postcolonial Predicaments and Deterritorialized Nation-States. New York: Routledge, 2003.

BASTIA, Tania. 'I Am Going, with or without You': Autonomy in Bolivian Transnational Migrations. Gender, Place \& Culture: A Journal of Feminist Geography, v. 20, n. 2, 2013, p. 160-177. 
BELVEDERE, Carlos; CAGGIANO, Sergio; CASARAVILLA, Diego et alii. Racismo y discurso: una semblanza de la situación argentina. In VAN DIJK, Teu (coord.). Racismo y discurso en América Latina. Barcelona: Gedisa, 2007, p. 35-88.

BENDINI, Mónica; BONACCORSI, Nélida (comps.). Con las puras manos: mujer y trabajo en regiones frutícolas de exportación. Buenos Aires: La Colmena, 1997.

BENDINI, Mónica; PESCIO, Cristina. Mujer y trabajo: las empacadoras de fruta del Alto Valle. In BENDINI, Mónica; BONACCORSI, Nélida (comps.). Con las puras manos: mujer y trabajo en regiones frutícolas de exportación. Buenos Aires: La Colmena, 1997, p. 15-30.

BENDINI, Mónica; RADONICH, Martha; STEIMBREGER, Norma. Nuevos espacios agrícolas, mercado de trabajo y migraciones estacionales. In RADONICH, Martha; STEIMBREGER, Norma (eds.). Reestructuraciones Sociales en Cadenas Agroalimentarias, GESA 6, Buenos Aires: La Colmena, 2007.

BENENCIA, Roberto. Bolivianización de la horticultura en la Argentina: procesos de migración transnacional y construcción de territorios productivos. In JELIN, Elizabeth; GRIMSON, Alejandro (comps.). Migraciones regionales hacia la Argentina: diferencia, desigualdad y derechos, Buenos Aires, Prometeo, 2005, p. 135-168.

BENENCIA, Roberto. Inserción de inmigrantes bolivianos y mercado de trabajo rural argentino. Familias transnacionales en la conformación de territorios productivos. Geodemos, n. 11, 2006, p. 17-38.

BENENCIA, Roberto; APARICIO, Susana (coords.). Nuevas formas de contratación en el trabajo agrario. Buenos Aires: CICCUS, 2014.

BENENCIA, Roberto; ATAIDE, Soraya. Segmented Labor Market and Migratory Identity Constructions in Two Horticultural Areas in the Province of Salta. In PIZARRO, Cynthia (comp.). Bolivian Labor Immigrants' Experiences in Argentina. Maryland: Lexington Books, 2016, p. 17-36.

BENENCIA, Roberto; QUARANTA, Germán; SOUZA CASADINHO, Javier (coords.). Cinturón hortícola de la Ciudad de Buenos Aires: cambios sociales y productivos. Buenos Aires: CICCUS, 2009.

BENENCIA, Roberto; RAMOS, Diego. Revisitando el cinturón hortícola de Río Cuarto, Córdoba: afianzamiento y cambios en el fenómeno migratorio de familias bolivianas. Río Cuarto: CEDERU-Facultad de Agronomía y Veterinaria de Río Cuarto, 2008.

BISIO, Raúl; FORNI, Floreal. Economía de enclave y satelización del mercado de trabajo rural. El caso de los trabajadores con empleo precario en un ingenio azucarero del noroeste argentino. Desarrollo Económico, v. 16, n. 61, 1976, p. 3-56.

BRAH, Avtar. Diferencia, diversidad, diferenciación. In HOOKS, Bell; BRAH, Avtar; SANDOVAL, Chela; ANZALDÚA, Gloria. Otras inapropiables: feminismos desde las fronteras. Madrid: Traficantes de Sueños, 2004, p. 107-136.

BRIONES, Claudia. La alteridad del" Cuarto Mundo": una deconstrucción antropológica de la diferencia. Buenos Aires: Ediciones del Sol, 1998.

BRIONES, Claudia. Cartografías argentinas: políticas indigenistas y formaciones provinciales de alteridad. Buenos Aires: Antropofagia, 2005. 
BRUNO, Sebastián. Inserción laboral de los migrantes paraguayos en Buenos Aires. Una revisión de categorías: desde el "nicho laboral" a la "plusvalía étnica". Población y Desarrollo, n. 3, 2008.

CAGGIANO, Sergio. Lo que no entra en el crisol. Inmigración boliviana, comunicación intercultural y procesos identitarios. Buenos Aires: Prometeo, 2005.

CIARALLO, Ana. Tensiones, resistencias y desigualdades en los nuevos escenarios de la horticultura en el norte de la Patagonia argentina. In TRPIN, Verónica; KREITER, Analía; BENDINI, Mónica (coords.). Abordajes interdisciplinarios en los estudios agrarios: desafíos de la investigación social en el norte de la Patagonia. General Roca: Publifadecs, 2013, p. 131-149.

CIARALLO, Ana; TRPIN, Verónica. Migration and Labor Market in Horticulture: Bolivian Families in the Middle Valley of the Río Negro, Argentine Patagonia. In PIZARRO, Cynthia (comp.). Bolivian Labor Immigrants' Experiences in Argentina. Maryland: Lexington Books, 2016, p. 1-16.

COMAROFF, Jean; COMAROFF, John. Home-made Hegemony: Modernity, Domesticity, and Colonialism in South Africa. New Jersey: Rutgers University Press, 1992.

CONTI, Viviana; LAGOS, Marcelo. Mano de obra indígena en los ingenios de Jujuy a principios de siglo. Buenos Aires: Centro Editor de América Latina, 1988.

CORTES, Genevieve. Migraciones, construcciones transnacionales y prácticas de circulación: un enfoque desde el territorio. Párrafos Geográficos, v. 8, n. 1, 2009, p. 35-53.

COURTIS, Corina; PACECCA, María Inés. Género y trayectoria migratoria: mujeres migrantes y trabajo doméstico en el Área Metropolitana de Buenos Aires. Papeles de Población, v. 63, n. 16, 2010, p. 155-185.

CRENSHAW, Kimberlé. Mapping the Margins: Intersectionality, Identity Politics, and Violence against Women of Color. In CRENSHAW, Kimberlé; GOTANDA, Neil et alii (eds.). Critical Race Theory. New York: The New Press, 1995.

DANDLER, Jorge; MEDEIROS, Carmen. Temporary Migration from Cochabamba, Bolivia to Argentina: Patterns and Impact in Sending Areas. In PESSAR, Patricia. When Borders Don't Divide: Labour Migration and Refugee Movements in the Americas. New York: Center for Migration Studies, 1988.

DUNCAN, Kenneth; RUTLEDGE, lan (comps.). La tierra y la mano de obra en América Latina. Ensayo sobre el desarrollo del capitalismo agrario en los siglos XIX y XX. México: Fondo de Cultura Económica, 1987.

FALQUET, Jules. La règle du jeu: repenser la co-formation des rapports sociaux de sexe, de classe et de «race» dans la mondialisation néolibérale. In DORLIN, Elsa (avec la collaboration d' Annie Bidet). Sexe, race, classe: pour une épistémologie de la domination. Paris: Actuel Marx Confrontation, 2009, p. 71-90.

FENTON, Steve. Ethnicity: Racism, Class and Culture. New York: Rowman \& Littlefield, 1999.

FONSECA, Claudia. Família, fofoca e honra. Etnografia de relações de gênero e violência em grupos populares. Porto Alegre: UFRGS Editora, 2004. 
FONSECA, Claudia. La clase social y su recusación etnográfica. Revista Etnografías Contemporáneas, año 1, n. 1, 2005, p. 117-138.

FORNI, Floreal; ROLDÁN, Laura. Trayectorias laborales de residentes de áreas urbanas pobres. Un estudio de casos en el conurbano bonaerense. Desarrollo EConómico, v. 35, n. 140, 1996, p. 585-599.

GARCÍA, Matías. Uso y acceso a la tierra en el marco del muevo modelo productivo de la horticultura platense. Revista Interdisciplinaria de Estudios Agrarios, n. 29, 2008, p. 79-98.

GERMANI, Gino. Emigración del campo a la ciudad y sus causas. In GIBERTI, Horacio; SOLARI, Aldo; GERMANI, Gino; OCHOA DE EGUILEOR, Jorge Ochoa de Eguileor. Sociedad, Economía y Reforma Agraria. Buenos Aires: Ediciones Libera, 1965, p. 71-87.

GIARRACCA, Norma; BIDASECA, Karina; MARIOTTI, Daniela. Tucumanos y tucumanas: zafra, trabajo, migraciones e identidad. Buenos Aires: Editorial La Colmena, 2000.

GIL HERNÁNDEZ, Franklin. Estado y procesos políticos. Sexualidad e interseccionalidad. In CORRÊA, Sonia; PARKER, Richard (orgs.). Sexualidade e política na América Latina: histórias, interseções e paradoxos. Rio de Janeiro: ABIA, 2011, p. 80-99.

GORDILLO, Gastón. Después de los ingenios: la mecanización de la zafra saltojujeña y sus efectos sobre los indígenas del Chaco centro-occidental. Desarrollo Económico, v. 35, n. 137, 1995, p. 105-126.

GRECO, María Gabriela. Alternativas laborales y movilidad espacial de la población: los cambios en la demanda de mano de obra por parte de la agroindustria azucarera y sus efectos socioespaciales. Ponencia presentada en el $6^{\circ}$ Encuentro de geógrafos de América Latina, 1997.

GRIMSON, Alejandro (comp.). Fronteras, naciones e identidades: la periferia como centro. Buenos Aires: La Crujía, 2000.

GRIMSON, Alejandro. Los límites de la cultura: crítica de las teorías de la identidad. Buenos Aires: Siglo XXI, 2011.

HAESBAERT, Rogerio. O mito da desterritorialização: do fim dos territórios à multiterritorialidade. Rio de Janeiro: Bertand Brasil, 2004.

HALPERN, Gerardo. Etnicidad, inmigración y política: representaciones y cultura política de exiliados paraguayos en Argentina. Buenos Aires: Prometeo, 2009.

HARVEY, David. El nuevo imperialismo: acumulación por desposesión. In PANTICH, Leo; LEYS, Colin (eds.). El Nuevo desafío Imperial. Buenos Aires: Merlin PressClacso, 2004, p. 99-129.

HOLMES, Seth. "Oaxacans Like to Work Bent Over": The Naturalization of Social Suffering among Berry Farm Workers. International Migration, v. 45, n. 3, 2007, p. 39-68.

JIMÉNEZ ZUNINO, Cecilia. Desclasamiento y reconversiones en las trayectorias de los migrantes argentinos de clases medias. Madrid: Universidad Complutense de Madrid, 2011. 
KARASIK, Gabriela. Migraciones, trabajo y corporeidad. Bolivianos y nativos en el trabajo rural y el servicio doméstico de Jujuy. In KARASIK, Gabriela (coord.). Migraciones internacionales: Reflexiones y estudios sobre la movilidad territorial contemporánea. Buenos Aires: CICCUS, 2013, p. 231-256.

MAGLIANO, María José. El género y la historia oral en los estudios sobre las migraciones internacionales: aportes y desafíos. Anuario de la Escuela de Historia, n. 22, 2009-2010, p. 81-99.

MAGLIANO, María José. Los significados de vivir "múltiples presencias": Mujeres bolivianas en Argentina. Migraciones Internacionales, v. 7, n. 24, 2013, p. 165-195.

MALLIMACI BARRAL, Ana. Nuevas miradas. Aportes de la perspectiva de género al estudio de los fenómenos migratorios. In COHEN, Néstor; MERA, Carolina (comps.). Relaciones interculturales: experiencias y representación social de los migrantes. Buenos Aires: Antropofagia, 2005, p. 115-138.

MALLIMACI BARRAL, Ana. Localizando el sentido de las desigualdades. Inclusiones y exclusiones de los/as bolivianos/as en Ushuaia. In KARASIK, Gabriela (coord.). Migraciones internacionales: reflexiones y estudios sobre la movilidad territorial contemporánea. Buenos Aires: CICCUS, 2013, p. 87-107.

MANCANO FERNANDES, Bernardo. Movimentos socioterritoriais e movimentos socioespaciais: contribuição teórica para uma leitura geográfica dos movimentos sociais. Revista Nera, n. 6, 2012, p. 24-34.

MANZANAL, Mabel. Regiones, territorios e institucionalidad del desarrollo rural. In MANZANAL, Mabel; NEIMAN, Guillermo; LATTUADA, Mario (comps.). Desarrollo rural: organizaciones, instituciones y territorio. Buenos Aires: CICCUS, 2006, p. 21-50.

MANZANAL, Mabel. Territorio, poder e instituciones: una perspectiva crítica. In MANZANAL, Mabel; ARQUEROS, María Ximena; NUSSBAUMER, Beatriz (comps.). Territorios en construcción: actores, tramas y gobiernos, entre la cooperación y el conflicto. Buenos Aires: CICCUS, 2007, p. 15-50.

MANZANAL, Mabel; ARQUEROS, María Ximena; ARZENO, Mariana; NARDI, María. Desarrollo territorial en el norte argentino. Una perspectiva crítica. Revista Eure, v. XXXV, n. 105, 2009, p. 131-153.

MARGULIS, Mario. La racialización de las relaciones de clase. In MARGULIS, Mario; URRESTI, Marcelo et alii. La segregación negada: cultura y discriminación social. Buenos Aires: Biblos, 1999, p. 37-62.

MASTRANGELO, Andrea; TRPIN, Verónica. Trabajo rural en producciones que Argentina exporta: una síntesis de características relevadas en estudios etnográficos recientes. Ponencia presentada en el VII Congresso Latino-americano de estudos do trabalho, San Pablo, 2013.

MELLIASOUX, Claude. Mujeres, graneros y capitales. Bogotá: Siglo XII, 1993.

MIRALLES, Glenda. Entre la casa y el galpón ihay lugar para el sindicato? General Roca: Publifadecs, 2004.

MIRALLES, Glenda; RADONICH, Martha. De trabajadoras familiares y asalariadas de los Valles de los ríos Negro y Neuquén. In MASSERONI, Susana; BENDINI, 
Mónica (comps.). El trabajo femenino: distintos ámbitos y abordajes. Documento de Trabajo n. 35. Buenos Aires: Instituto de Investigaciones Gino Germani, Facultad de Ciencias Sociales, Universidad de Buenos Aires, 2003, p. 69-81.

MORBERG, Mark. Myth of Ethnicity and Nation: Immigration, Work and Identity in the Belize Banana Industry. Knoxville: University of Tennesee Press, 1997.

NEIMAN, Guillermo. Los estudios sobre el trabajo agrario en la última década: una revisión para el caso argentino. Mundo Agrario, v. 10, n. 20, 2010.

ORTNER, Sherry. La teoría antropológica desde los años sesenta. Guadalajara: Universidad de Guadalajara, 1993.

PARELLA RUBIO, Sonia. El trasvase de desigualdades de clase y etnia entre mujeres de servicios de proximidad. Papers, n. 60, 2000, p. 275-289.

PEDONE, Claudia. Varones aventureros vs. madres que abandonan: reconstrucción de las relaciones familiares a partir de la migración ecuatoriana. REMHU, Revista Interdisciplinar da Mobilidade Humana, v. 16, n. 30, 2008, p. 45-64.

PEDREÑO CÁNOVAS, Andrés. Sociedades etnofragmentadas. In PEDREÑO CÁNOVAS, Andrés; HERNÁNDEZ PEDREÑO, Manuel (coords.). La condición inmigrante. Exploraciones e investigaciones desde la Región de Murcia. Murcia: Universidad de Murcia, 2005, p. 75-106.

PENA, Mariela. Aportes de la incorporación de perspectivasfeministas a las investigaciones con técnicas orales. Ponencia presentada en las X Jornadas de Sociología. Buenos Aires: Facultad de Ciencias Sociales, Universidad de Buenos Aires, 2013.

PÉREZ, Natalia Evangelina. "Quiero que mis hijos estudien, para que no trabajen a pico y pala como nosotros": prácticas escolares de marcación y (des)marcación de la etnicidad boliviana. In KARASIK, Gabriela (ed.). Migraciones internacionales. Reflexiones y estudios sobre la movilidad territorial contemporánea. Buenos Aires: CICCUS, 2013, p. 198-208.

PIZARRO, Cynthia. Espacios socioculturales "bolivianos" trans-urbanos en el Área Metropolitana de Buenos Aires. In MARONESE, Leticia (ed.). Buenos Aires Boliviana. Migración, construcciones identitarias y memoria. Buenos Aires: Ministerio de Cultura, Gobierno de la Ciudad Autónoma de Buenos Aires, 2009, p. 37-52.

PIZARRO, Cynthia (coord.). Migraciones internacionales contemporáneas: estudios para el debate. Buenos Aires: CICCUS, 2011.

PIZARRO, Cynthia. Sufriendo y resistiendo la segregación laboral: experiencias de inmigrantes bolivianos que trabajan en el sector hortícola de la región metropolitana de la ciudad de Córdoba. In PIZARRO, Cynthia (coord.). Migraciones internacionales contemporáneas: estudios para el debate. Buenos Aires: CICCUS, 2011, p. 335-358.

PIZARRO, Cynthia (ed.). "Ser boliviano" en la región metropolitana de la ciudad de Córdoba. Localización socio-espacial, mercado de trabajo y relaciones interculturales. Córdoba: Editorial de la Universidad Católica de Córdoba, 2011.

PIZARRO, Cynthia. Sanidad, calidad: bioregulación y disciplinamiento. Las buenas prácticas agrícolas en la producción hortícola argentina. Revista Ruris, v. 6, n. 2, 2012, p. 155-180. 
PIZARRO, Cynthia. Clasificar a los otros migrantes: las políticas de migración argentinas como productoras de etnicidad y desigualdad. Revista Métis, Historia \& Cultura, v. 11, n. 22, 2012, p. 219-240.

PIZARRO, Cynthia. Discurso racializante y segmentación étnico-nacional del mercado laboral: Trabajadores bolivianos en un cortadero de ladrillos de Córdoba, Argentina. In BENENCIA, Roberto; HERRERA LIMA, Fernando; LEVINE, Elaine (coords.). Ser migrante latinoamericano, ser vulnerable, trabajar precariamente. Madrid: Editorial de la División de Ciencias Sociales y Humanidades, Universidad Autónoma Metropolitana-Campus Iztapalapa - Editorial Anthropos, 2012, p. 79-94.

PIZARRO, Cynthia. La bolivianidad en disputa. (Des)marcaciones de etnicidad en contextos migratorios. In KARASIK, Gabriela (coord.). Migraciones internacionales. Reflexiones y estudios sobre la movilidad territorial contemporánea. Buenos Aires: CICCUS, 2013, p. 331-360.

PIZARRO, Cynthia. Partir y volver entre Argentina y Bolivia: trayectorias migratorias transnacionales intersección de desigualdades. Ponencia presentada en las VII Jornadas Santiago Wallace de Investigación en Antropología Social, Universidad de Buenos Aires, 2013.

PIZARRO, Cynthia. (ed.). Bolivianos y bolivianas en la vida cotidiana cordobesa: Trabajo, derechos e identidad en contextos migratorios. Córdoba: Editorial de la Universidad Católica de Córdoba, 2015.

PIZARRO, Cynthia. Intersection of Inequalities: Migratory Trajectories, Labor Experiences, and Family Life of Bolivian Women on the Outskirts of Buenos Aires and Córdoba. In PIZARRO, Cynthia (ed.). Bolivian Labor Immigrants' Experiences in Argentina. Maryland: Lexington Books, 2016, p. 51-66.

PIZARRO, Cynthia. (ed.). Bolivian Labor Immigrants' Experiences in Argentina. Maryland: Lexington Books, 2016.

PIZARRO, Cynthia. Trabajadores paraguayos en la producción forestal del Delta Inferior del río Paraná. In APARICIO, Susana; BENENCIA, Roberto (eds.). De migrantes y asentados. Trabajo estacional en el agro argentino. Buenos Aires: CICCUS, 2016, p. 112-136.

PIZARRO, Cynthia; BASUALDO, María Lourdes; FERREIRO, Mariana. Practices of Resistance among Young Bolivian Immigrants Working in a Brick Factory in Córdoba. In PIZARRO, Cynthia (ed.). Bolivian Labor Immigrants' Experiences in Argentina. Maryland: Lexington Books, 2016, p. 85-104.

PIZARRO, Cynthia; FABBRO, Pablo; FERREIRO, Mariana. "No conozco trabajo más perro que éste": Reproducción y resistencia de la subalternidad de los trabajadores bolivianos en un cortadero de ladrillos de la ciudad de Córdoba, Argentina. In BETRISEY NADALI, Débora (comp.). Poder, Políticas e Inmigración en América Latina. Barcelona: Editorial Bellaterra, 2014, p. 95-112.

PIZARRO, Cynthia; MORENO, Marta Silvia. Differential Migration Pathways of Bolivian Women Working in Horticultural Fields in Mendoza. In PIZARRO, Cynthia (ed.). Bolivian Labor Immigrants' Experiences in Argentina. Maryland: Lexington Books, 2016, p. 37-50. 
PIZARRO, Cynthia; TRPIN, Verónica. Trabajadores frutícolas y hortícolas en la Argentina: una aproximación socioantropológica a las prácticas de reproducción y de resistencia de las condiciones laborales. RURIS, v. 4, n. 2, 2010, p. 199-228.

QUARANTA, Germán. Estructura ocupacional, características de la demanda y perfil de la oferta laboral en el agro argentino a principios de la década actual. In NEIMAN, Guillermo (dir.). Estudios sobre la demanda de trabajo en el agro argentino. Buenos Aires: CICCUS, 2010, p. 62-92.

QUARANTA, Germán; FABIO, Francisco. Intermediación laboral y mercados de trabajo en agriculturas reestructuradas: el caso del Valle de Uco, Mendoza, Argentina. Región y Sociedad, v. XXIII, n. 51, 2011, p. 193-225.

QUIJANO, Aníbal. Colonialidad del poder, eurocentrismo y América Latina. In LANDER, Edgardo (comp.). La colonialidad del saber: eurocentrismo y ciencias sociales. Perspectivas Latinoamericanas. Buenos Aires. CLACSO, 2000, p. 201-246.

RADONICH, Martha. Territorio, migración y trabajo en la fruticultura del norte de la Patagonia. Tesis de Doctorado. Universidad de Murcia, España. Tesis no publicada, 2010.

RADONICH, Martha; CIARALLO, Ana; TRPIN, Verónica. Chilenos y bolivianos en la conformación de territorios en áreas rurales del Alto Valle de Río Negro, Argentina. In PIZARRO, Cynthia (coord.). Migraciones internacionales contemporáneas: estudios para el debate. Buenos Aires: CICCUS, 2011, p. 379-400.

RADONICH, Martha; TRPIN, Verónica. Mujeres migrantes en la organización de territorios rurales en el Alto Valle de Río Negro. In KARASIK, Gabriela (coord.). Migraciones internacionales: reflexiones y estudios sobre la movilidad territorial contemporánea. Buenos Aires: CICCUS, 2013, p. 279-304.

RAFFESTIN, Claude. Por uma geografia do poder. São Paulo: Ática, 1993.

RATIER, Hugo. El cabecita negra. Buenos Aires: Centro Editor de América Latina, 1972.

REBORATTI, Carlos. El territorio rural: zactor o escenario? Ponencia presentada en las $V$ Jornadas de investigación y debate sobre: Trabajo, propiedad y tecnología en el mundo rural argentino. Bernal: Universidad Nacional de Quilmes, 2008.

RIVERO SIERRA, Fulvio. Condiciones de emigración y cultura migratoria en dos casos de Bolivia: Toropalca en Potosí y B Plan 3000 en Santa Cruz de la Sierra. Actas del Tercer Congreso Latinoamericano de Antropología ALA, Santiago de Chile, 2012.

ROFMAN, Alejandro. Economías regionales extrapampeanas y exclusión social en el marco del ajuste. EURE (Santiago), v. 23, n. 70, 1997, p. 19-37.

ROSAS, Carolina. Dilemmas and Decisions of Peruvian Couples before Migrating to Argentina. Ponencia presentada en 74th Annual Meeting Society for Applied Anthropology, 2014.

SANTOS, Milton. Espacio y método. Barcelona: Universidad de Barcelona, 1986.

SASSONE, Susana. Breve geografía histórica de la migración boliviana en la Argentina. In MARONESE, Leticia (ed.). Buenos Aires Boliviana. Migración, construcciones identitarias y memoria. Buenos Aires: Ministerio de Cultura, Gobierno de la Ciudad Autónoma de Buenos Aires, 2009, p. 389-402. 
SAYAD, Abdelmalek. A imigração ou os paradoxos de alteridade. São Paulo: Editora da Universidade de São Paulo, 1998.

SILI, Marcelo. La Argentina Rural. De la crisis de la modernización agraria a la construcción de un nuevo paradigma de desarrollo de los territorios rurales. Buenos Aires: Ediciones INTA, 2005.

SILVERSTEIN, Paul. Immigrant Racialization and the New Savage Slot: Race, Migration, and Immigration in the New Europe. Annual Review of Anthropology, 2005, p. 363-384.

SOCHACZEWSKI, Evelyn. Y salió a vender el día. Los desconocidos migrantes temporarios urbanos. Ponencia presentada en Se fue a volver. Seminario sobre migraciones temporales en América Latina. PISPAL/CIUDAD/CENEP, 1986.

STEIMBREGER, Norma; TRPIN, Verónica; BENDINI, Mónica. Intermediación laboral en el acceso y gestión del trabajo estacional en la fruticultura rionegrina. Revista Interdisciplinaria de Estudios Agrarios, n. 37, 2012, p. 5-30.

TARRIUS, Alain. Leer, describir, interpretar. Las circulaciones migratorias: conveniencia de la noción de "territorio circulatorio". Los nuevos hábitos de la identidad. Relaciones, v. XXI, n. 83, 2000, p. 39-66.

TRINCHERO, Hugo. Los dominios del demonio. Civilización y barbarie en las fronteras de la nación. El chaco central. Buenos Aires: EUDEBA, 2000.

TRPIN, Verónica. Aprender a chilenos: identidad, trabajo y residencia de familias migrantes en el Alto Valle de Río Negro. Buenos Aires: Antropofagia, 2004.

TRPIN, Verónica; BROUCHOUD, Silvia. Mujeres migrantes en producciones agrarias de Río Negro: aportes para abordar la interseccionalidad en las desigualdades. Párrafos geográficos, v. 13, n. 2, 2014, p. 108-126.

VÁZQUEZ LABA, Vanesa. Re-pensando la división sexual del trabajo familiar: aspectos teóricos y empíricos para la interpretación de los modelos de familia en el noroeste argentino. Trabajo y Sociedad, v. 10, n. 11, 2008.

WIEVIORKA, Michel. Diferencias culturales, racismo y democracia. In MATO, Daniel (coord.). Políticas de identidades y diferencias sociales en tiempos de globalización. Caracas: FACES/ UCV, 2003, p. 17-32.

WOLF, Eric. Europa y la gente sin historia. México: Fondo de Cultura Económica, 1987.

Recibido para publicación en 24.01.2017

Aceptado para publicación en 06.03.2017

Received for publication in January $24^{\text {th }}, 2017$

Accepted for publication in March 06 ${ }^{\text {th }}, 2017$

ISSN impresso 1980-8585

ISSN eletrônico 2237-9843

http://dx.doi.org/10.1590/1980-85852503880004903 